\title{
WASPAS Method for Defining a Content Creator
}

\author{
Muhammad Sabir Ramadhan ${ }^{\mathrm{a}}$, Nizwardi Jalinus ${ }^{\mathrm{b}}$, Refdinal ${ }^{\mathrm{c}}$, SaifulNurArif ${ }^{\mathrm{d}}$, MukhlisRamdhan ${ }^{\mathrm{e}}$, \\ Muhammad Dahria ${ }^{f}$, Asyahri Hadi Nasyuha ${ }^{g}$
}

${ }^{\text {a, }}$ Faculty of Engineering, Asahan University, Kisaran, Indonesia

${ }^{\mathrm{b}}$ Faculty of Engineering, Universitas Negeri Padang, Indonesia

${ }^{c}$ Faculty of Engineering, Universitas Negeri Padang, Indonesia

${ }^{\mathrm{d}}$ Computer System of STMIK Triguna Dharma, Medan, Indonesia

${ }^{\mathrm{e},}$ Computer System of STMIK Triguna Dharma, Medan, Indonesia

f. Information System of STMIK Triguna Dharma, Medan, Indonesia

g, Information System of STMIK Triguna Dharma, Medan, Indonesia

aramasabir@gmail.com,jnizwardi228@gmail.com, ${ }^{\mathrm{c}}$ refmoein@gmail.com, ${ }^{\mathrm{d}}$ Saiful.nurarief@ gmail.com, emukhlis_ramadhan@trigunadharma.ac.id, ${ }^{\mathrm{f}} \mathrm{m}$ _dahria@trigunadharma.ac.id, ${ }^{\mathrm{g}}$ asyahrihadi@gmail.com

\begin{abstract}
In the era of the Industrial Revolution 4.0, social media is very influential in marketing company products, therefore companies interested in using social media need the role of content creators and employees. This is because the selection of prospective employees for content creators is still manual, and the selection of employees who are still related to family leads to an objective selection. Information technology and decision support systems need to be used as a tool to determine the selection of quality content creation staff using the WASPAS method, a combination of the WSM and WPM methods. By assigning weights to each criterion and then conducting a ranking evaluation process, this method can be used to solve the "multi-criteria decision making" problem. The result of this research is to find content creators who meet the standards of the WASPAS analysis method.
\end{abstract}

KEYWORDS: Content Creator;Decision Support System;WASPAS.

\section{INTRODUCTION}

In the era of the Industrial Revolution 4.0, social media is currently very influential(Daniel et al., 2018)(Salman et al., 2016), and many companies are interested in using social media as a way to market products(McShane et al., 2019)(McShane et al., 2019), so it takes creative people to come. Create content, but what is happening now is that the content obtained is not in accordance with the company's wishes(McShane et al., 2019). The problem of content creator employees is due to the manual recruitment process and the selection of employees who are still connected with their families. Employee records itself is a process of finding, finding and attracting job applicants in a company or organization (Setiani, 2013). Therefore, information technology and decision support systems are needed as a tool to determine the selection of quality content creator employees. Like previous studies, a decision support system is a system specifically designed for semi-structured and unstructured problem decision-making processes. In order to properly achieve the objectives of the DSS, a method in the decision support system (namely the WASPAS method) can be used to assist this method, which is a combination of the WSM and WPM methods. This method can be used to solve MCDM (multi-criteria decision-making) problems. Previous research has proven that by using the WASPAS method to determine the weighted value of each criterion, then through the ranking process prospective employees can select prospective employees who meet the requirements. (Handayani et al., 2018)(Sugiarti et al., 2018)(Safrizal et al., 2018).

\section{METHODOLOGY}

Decision Support System (DSS) is an interactive information system used to provide information, modeling, and data processing. This system functions in making decisions in both semistructured and unstructured situations(Kusrini M.Kom, n.d.)(Purwokerto et al., 2014)(Zavadskas et al., 2012). The Decision Support System states that the Decision Support System is a system that helps make decisions using data and models(Abdullah \& Adawiyah, 2014). A system that can solve problems (Al-hafiz, 2017)(Nasyuha, 2019)(Gunawan, 2015)(Hossein Hojjati \& Anvary, 2013). Decision Support Systems are computer-based information systems capable of 
providing information, modeling and manipulating data. This system is used for a problem in decision making in a semi-structured and unstructured situation. Basically, a Decision Support System is designed to support all stages of decision making, from identifying problems, selecting relevant data, determining the approach used in the decision-making process, to evaluating alternative choices. Decision support system (DSS) is part of a computerbased information which includes a knowledge-based system or knowledge management that is used as decision support in an organization or company.Decision Support Systems have the following objectives, to assist managers in making decisions to solve a structured problem. support the manager's judgment in making decisions and not try to replace the manager's position, increasing the effectiveness of manager's decision-making rather than its efficiency. In Decision Making, there are three stages that must be passed as follows:

a. The tracing and detection stage of the problematic scope is in the process of problem recognition, the incoming data is processed and tested in order to identify the problem.

b. The design stage is the process of tracing, developing, and analyzing alternative actions that can be taken.

c. The stage of selecting among various alternative actions that may be carried out. The results are then implemented in a decision-making process.

Conceptually, there are three elements related to the Decision Making System, namely:

a. Problems

In a decision support system there are several types of problems, namely: Structured Problems, Semitructured Problems, Unstructured Problems.

b. Solutions

In solving a problem, the Decision Support System has several types of solutions, namely: Multi Attribute Decision Making (MADM), Simple Additive Weighting (SAW) Method, Analytical Hierarchy Process (AHP) Method, Weighted Aggregated Sum Production Assessment (WASPAS) Method.

c. Results

The result or output of a Decision Support System is in the form of a decision from a problem being researched or discussed which can be used as a benchmark for a policy.

In weighting, the weight is the value or value of a criterion indicator. There are things that need to be considered in the weighting of a decision support system, namely, the weighting sources of the criteria, the sub-criteria (indicators) the causes of a problem being discussed must come from the optional standards (standard standards) and the policy makers from the case studies (case studies) discussed. .

There are several rules for weighting criteria in a decision support system, namely:

a. The percentage approach. Has a value range of 0 to $100 \%$ with a record value of $\sum \mathrm{Wj}=100 \%$

b. 2. Fuzzy Logic Approach. Has a value range of 0 to 1

c. 3. Actual Value Approach. Has a value range of 0 to 10 or 0 to 100 with normalization $\sum \mathrm{Wj}=100 \%$ except for the Profile Matching method which has an sktusl value from 0 to 5

\subsection{Weighted Aggregated Sum Product Assessment}

Weighted Aggregated Sum Product Assessment (WASPAS) is a method that has an error rate which is a combination method from two sources commonly known as WMM, MCDM (Multi Criteria Decision Making) approaches and WPM (Weigth Product Models). The WASPAS method is a method used to reduce errors and determine estimates when selecting the highest and lowest scores. The WASPAS method is a unique combination of the WSM and WPM methods. WASPAS is used to solve various problems, such as decision making, evaluation, alternatives, etc[9][8][18]. Following are the steps that must be completed using the Weighted Sum Product Evaluation (WASPAS) method, namely(Hutagalung et al., 2018):

1. First, make a value for each alternative (Xij) and on each criterion (Cij) that has been determined(Sianturi et al., 2018)(Amalia et al., 2019).

2. Make a decision matrix

$$
X=\left(\begin{array}{llll}
X_{11} & X_{12} & \ldots & X_{1 n} \\
X_{21} & X_{22} & \ldots & X_{2 n} \\
X_{m 1} & X_{m 2} & \ldots & X_{m n}
\end{array}\right)
$$

3. Normalizing the $\mathrm{X}$ matrix. For Benefit Criteria (Handayani \& Marpaung, 2018):

$$
\mathrm{X}_{\mathrm{ij}}=\frac{x_{i j}}{\max i x_{i j}}
$$

For Cost Criteria 


$$
\mathrm{X}_{\mathrm{ij}}=\frac{\min _{i x i j}}{x i j}
$$

4. Calculating the Preference Value $\left(\mathrm{Q}_{\mathrm{i}}\right)($ Panjaitan et al., 2019):

$$
Q i=0,5 \sum_{j=1}^{n} X i j w+0,5 \prod_{j=1}^{n}(x i j)^{w j}
$$

Where :

$\mathrm{Qi}=$ Value from $\mathrm{Q}$ to $\mathrm{i}$

Xij $\mathrm{W}=$ Multiply the value of Xij by weight $(\mathrm{w})$

$0.5=$ Provision

The alternative that has the highest Qi value is the best alternative.

\section{EXPERIMENTAL RESULT}

The following research methods used in this study are(Indriani \& Warnilah, 2019):

1. Observation

In making observations, it is reviewed directly to companies that need a content creator. In these companies, analysis and observations are carried out how the previous system was in the selection of content creator employees, where the system there is still manual in selecting content creator employees.

2. Interview

After making observations, interviews are conducted with company leaders or related parties who need a system in selecting content creator employees. In Table 1. below shows the data obtained from companies that require content creators with the criteria used, the data are as follows:

\begin{tabular}{|c|c|c|c|c|c|c|c|c|}
\hline \multirow{2}{*}{ No } & \multirow{2}{*}{$\begin{array}{c}\text { Alterna } \\
\text { tive }\end{array}$} & \multicolumn{7}{|c|}{ Criteria } \\
\hline & & C1 & $\mathrm{C2}$ & C3 & $\mathrm{C4}$ & C5 & C6 & C7 \\
\hline 1 & $\begin{array}{c}\text { Melinda } \\
\text { Gina } \\
\text { Tresia }\end{array}$ & $\begin{array}{l}\text { Pretty } \\
\text { good }\end{array}$ & $\begin{array}{c}\text { Master } \\
\text { CorelDraw }\end{array}$ & $\begin{array}{l}\text { Master Corel } \\
\text { Video Studio }\end{array}$ & $\begin{array}{c}2 \\
\text { Certificates }\end{array}$ & $\begin{array}{c}6 \\
\text { Months }\end{array}$ & Diploma 3 & $\begin{array}{l}\text { Pretty } \\
\text { Good }\end{array}$ \\
\hline 2 & $\begin{array}{c}\text { Andika } \\
\text { Chambe } \\
\text { rlin }\end{array}$ & Good & $\begin{array}{c}\text { Master } \\
\text { CorelDraw }\end{array}$ & $\begin{array}{c}\text { Master } \\
\text { Cyberlink } \\
\text { Power Director }\end{array}$ & $\begin{array}{c}4 \\
\text { Certificates }\end{array}$ & 2 Years & $\begin{array}{c}\text { vocational } \\
\text { high } \\
\text { School } \\
\end{array}$ & Good \\
\hline 3 & $\begin{array}{l}\text { Andrian } \\
\text { syah }\end{array}$ & $\begin{array}{l}\text { Very } \\
\text { Good }\end{array}$ & $\begin{array}{c}\text { Master Adobe } \\
\text { Photohop }\end{array}$ & $\begin{array}{l}\text { Master Corel } \\
\text { Video Studio }\end{array}$ & $\begin{array}{c}6 \\
\text { Certificates }\end{array}$ & 2 Years & $\begin{array}{l}\text { Senior } \\
\text { High } \\
\text { School }\end{array}$ & Good \\
\hline 4 & $\begin{array}{l}\text { Naufal } \\
\text { Alfaqir }\end{array}$ & $\begin{array}{l}\text { Very } \\
\text { Good }\end{array}$ & $\begin{array}{c}\text { Master } \\
\text { CorelDraw, } \\
\text { dan Adobe } \\
\text { Photohop }\end{array}$ & $\begin{array}{c}\text { Master Corel } \\
\text { Video Studio, } \\
\text { Cyberlink } \\
\text { PowerDirector } \\
\text { dan Adobe } \\
\text { Primiere Pro }\end{array}$ & $\begin{array}{c}15 \\
\text { Certificates }\end{array}$ & $\begin{array}{c}3,5 \\
\text { Years }\end{array}$ & $\begin{array}{l}\text { vocational } \\
\text { high } \\
\text { School }\end{array}$ & $\begin{array}{l}\text { Very } \\
\text { Good }\end{array}$ \\
\hline 5 & $\begin{array}{c}\text { Apri } \\
\text { Manulla } \\
\text { ng }\end{array}$ & $\begin{array}{l}\text { Very } \\
\text { Good }\end{array}$ & $\begin{array}{c}\text { Master Adobe } \\
\text { Photoshop }\end{array}$ & $\begin{array}{l}\text { Master Adobe } \\
\text { Primiere Pro }\end{array}$ & $\begin{array}{c}5 \\
\text { Certificates }\end{array}$ & 3 Years & Diploma 1 & Good \\
\hline 6 & $\begin{array}{c}\text { Sri } \\
\text { Wahyun } \\
\text { i }\end{array}$ & Good & $\begin{array}{c}\text { Master Adobe } \\
\text { Photoshop }\end{array}$ & $\begin{array}{c}\text { Master } \\
\text { Cyberlink } \\
\text { Power Director }\end{array}$ & $\begin{array}{c}2 \\
\text { Certificates }\end{array}$ & $\begin{array}{c}6 \\
\text { Month }\end{array}$ & $\begin{array}{l}\text { Vocational } \\
\text { high } \\
\text { School } \\
\end{array}$ & Good \\
\hline 7 & $\begin{array}{c}\text { Saiful } \\
\text { Silungli } \\
\text { ngga }\end{array}$ & $\begin{array}{l}\text { Very } \\
\text { Good }\end{array}$ & $\begin{array}{c}\text { Master Adobe } \\
\text { Photoshop }\end{array}$ & $\begin{array}{l}\text { Master Corel } \\
\text { Video Studio }\end{array}$ & $\begin{array}{c}2 \\
\text { Certificates }\end{array}$ & $\begin{array}{c}2 \\
\text { Month }\end{array}$ & $\begin{array}{l}\text { Senior } \\
\text { High } \\
\text { School }\end{array}$ & $\begin{array}{l}\text { Very } \\
\text { Good }\end{array}$ \\
\hline 8 & $\begin{array}{c}\text { Dedy } \\
\text { Syaputr } \\
\text { a }\end{array}$ & $\begin{array}{l}\text { Not } \\
\text { Good }\end{array}$ & $\begin{array}{c}\text { Master Adobe } \\
\text { Photoshop }\end{array}$ & $\begin{array}{c}\text { Master } \\
\text { Cyberlink } \\
\text { Power Director }\end{array}$ & $\begin{array}{c}10 \\
\text { Certificates }\end{array}$ & 2 Years & $\begin{array}{l}\text { Vocational } \\
\text { high } \\
\text { School } \\
\end{array}$ & $\begin{array}{l}\text { Not } \\
\text { Good }\end{array}$ \\
\hline 9 & $\begin{array}{l}\text { Febbry } \\
\text { Anto } \\
\text { Situmor } \\
\text { ang }\end{array}$ & Good & $\begin{array}{c}\text { Master } \\
\text { CorelDraw }\end{array}$ & $\begin{array}{c}\text { Master } \\
\text { Cyberlink } \\
\text { Power Director }\end{array}$ & $\begin{array}{c}5 \\
\text { Certificates }\end{array}$ & $\begin{array}{c}6 \\
\text { Month }\end{array}$ & $\begin{array}{l}\text { Vocational } \\
\text { high } \\
\text { School }\end{array}$ & Good \\
\hline 10 & $\begin{array}{c}\text { Evi } \\
\text { Ulina } \\
\text { Sitorus }\end{array}$ & $\begin{array}{l}\text { Very } \\
\text { Good }\end{array}$ & $\begin{array}{c}\text { Master Adobe } \\
\text { Photoshop }\end{array}$ & $\begin{array}{l}\text { Master Corel } \\
\text { Video Studio }\end{array}$ & $\begin{array}{c}5 \\
\text { Certificates }\end{array}$ & 1 Year & Diploma 2 & $\begin{array}{l}\text { Very } \\
\text { Good }\end{array}$ \\
\hline
\end{tabular}

Table 1. Prospective Content Creator Employee Data from the Company 
Table 2. below explains that the decision-making process requires criteria that have been determined by the company in determining potential content creators, here are the criteria used:

Table 2. Name of Criteria and Weighted Criteria

\begin{tabular}{cclccc}
\hline No & Id & \multicolumn{1}{c}{ Criteria } & $\begin{array}{c}\text { Weight } \\
\left(\mathbf{W}_{\mathbf{j})}\right.\end{array}$ & Type \\
\hline 1 & $\mathrm{C}_{1}$ & Ethics & $20 \%=0,2$ & Benefit \\
\hline 2 & $\mathrm{C}_{2}$ & Mastery of Graphic Design Editing & $20 \%=0,2$ & Benefit \\
\hline 3 & $\mathrm{C}_{3}$ & Video Editing Mastery & & $20 \%=0,2$ & Benefit \\
\hline 4 & $\mathrm{C}_{4}$ & $\begin{array}{l}\text { Design Certificate } \\
\text { Grafis/Videografi }\end{array}$ & Charter & $5 \%=0,05$ & Benefit \\
\hline 5 & $\mathrm{C}_{5}$ & Work experience & & \\
\hline 6 & $\mathrm{C}_{6}$ & Education & $5 \%=0,05$ & Benefit \\
\hline 7 & $\mathrm{C}_{7}$ & Interview & $10 \%=0,1$ & Benefit \\
\hline
\end{tabular}

Based on the data that has been obtained, conversion of each criterion is carried out so that the calculation process can be carried out into the WASPAS method. The following is a conversion table of the criteria used:

a. Ethics

Table 3. below explains the weight value of the Ethical criteria as follows:

Table 3. Ethical criteria

\begin{tabular}{cc}
\hline Ethical Information & Alternative Weights \\
\hline Very Good & 5 \\
\hline Good & 4 \\
\hline Pretty Good & 3 \\
\hline Not Good & 2 \\
\hline Very Bad & 1 \\
\hline
\end{tabular}

b. Mastery of Graphic Design Editing

The weight value of the criteria for Graphic Design Editing Mastery is described in table 4. as follows:

Table 4. Graphic Design Editing Mastery

\begin{tabular}{lc}
\hline \multicolumn{1}{c}{ Software mastered in Graphic Editing } & Alternative Weights \\
\hline Master CorelDraw, Adobe Photoshop, dan Adobe Illustrator & 5 \\
\hline Master CorelDraw dan Adobe Photoshop & 4 \\
\hline Master Adobe Illustrator & 3 \\
\hline Master Adobe Photoshop & 2 \\
\hline Master CorelDraw & 1 \\
\hline
\end{tabular}

c. Video Editing Mastery

Table 5 explains the weight value of the Video Editing Mastery criteria as follows:

Table 5. Video Editing Mastery Criteria

\begin{tabular}{lc}
\hline \multicolumn{1}{c}{ Software mastered in Graphic Editing } & Alternative Weights \\
\hline $\begin{array}{l}\text { Master Corel Video Studio, Cyberlink PowerDirector dan Adobe } \\
\text { Primiere Pro }\end{array}$ & 5 \\
\hline Master Corel Video Studio dan Cyberlink PowerDirector & 4 \\
\hline Master Adobe Primiere Pro & 3 \\
\hline Master Cyberlink PowerDirector & 2 \\
\hline Master Corel Video Studio & 1 \\
\hline
\end{tabular}

d. Design Certificate / Charter Grafis/Videografi

The following is the weight value of the Graphic Design / Video Certificate / Charter criteria shown in table 6. as follows:

Table 6. Criteria for Certificate / Charter in the Field of Graphic / Video Design

\begin{tabular}{cc}
\hline Applicant's Owned Certificate & Alternative Weights \\
\hline $14-16$ Certificates & 5 \\
\hline $11-13$ Certificates & 4 \\
\hline
\end{tabular}




\begin{tabular}{ll}
\hline 8-10 Certificates & 3 \\
\hline 5-7 Certificates & 2 \\
\hline $0-4$ Certificates & 1 \\
\hline
\end{tabular}

e. Work Experiences

In table 7 . below the weight value of the work experience criteria is explained as follows:

Table 7. Work Experience

\begin{tabular}{lc}
\hline Work Experience & Alternative Weights \\
\hline 4 Years & 5 \\
\hline $3-4$ Years & 4 \\
\hline $2-3$ Years & 3 \\
\hline $1-2$ Years & 2 \\
\hline $0-1$ Years & 1 \\
\hline
\end{tabular}

f. Education

The following is the weight value of the education criteria which can be seen in table 8 . Below:

Table 8. Educational Criteria

\begin{tabular}{cc}
\hline Education & Alternative Weights \\
\hline Diploma 3 & 5 \\
\hline Diploma 2 & 4 \\
\hline Diploma 1 & 3 \\
\hline vocational high School & 2 \\
\hline Senior High School & 1
\end{tabular}

g. Interview

In table 9. the following is the weight value of the interview criteria as follows:

Table 9. Interview Criteria.

\begin{tabular}{cc}
\hline Results of the Interview & Alternative Weights \\
\hline Very Good & 5 \\
\hline Good & 4 \\
\hline Pretty Good & 3 \\
\hline Not Good & 2 \\
\hline Very Bad & 1 \\
\hline
\end{tabular}

Based on the data above, it is necessary to evaluate each criterion with a criteria table in order to perform calculations. In table 10 , the following is the data conversion result of alternative data.

Table 10. Results of alternative data conversion

\begin{tabular}{clccccccc}
\hline \multirow{2}{*}{ No } & \multirow{2}{*}{ Alternative } & \multicolumn{7}{c}{ Criteria } \\
\cline { 2 - 9 } & & $\mathbf{C}_{\mathbf{1}}$ & $\mathbf{C}_{\mathbf{2}}$ & $\mathbf{C}_{\mathbf{3}}$ & $\mathbf{C}_{\mathbf{4}}$ & $\mathbf{C 5}$ & $\mathbf{C 6}$ & $\mathbf{C}_{\mathbf{7}}$ \\
\hline 1 & Melinda Gina Tresia & 3 & 1 & 1 & 1 & 1 & 5 & 3 \\
\hline 2 & Andika Chamberlin & 4 & 1 & 2 & 1 & 1 & 2 & 4 \\
\hline 3 & Andriansyah & 5 & 2 & 1 & 2 & 2 & 1 & 4 \\
\hline 4 & Naufal Alfaqir & 5 & 4 & 5 & 5 & 4 & 2 & 5 \\
\hline 5 & Apri Manullang & 5 & 2 & 3 & 2 & 4 & 3 & 4 \\
\hline 6 & Sri Wahyuni & 4 & 2 & 2 & 1 & 1 & 2 & 4 \\
\hline 7 & Saiful Silunglingga & 5 & 2 & 1 & 1 & 1 & 1 & 5 \\
\hline 8 & Dedy Syaputra & 2 & 2 & 2 & 3 & 2 & 2 & 2 \\
\hline 9 & Febbry Anto Situmorang & 4 & 1 & 2 & 2 & 1 & 2 & 4 \\
\hline 10 & Evi Ulina Sitorus & 5 & 2 & 1 & 2 & 1 & 4 & 5 \\
\hline & Nilai Max & 5 & 4 & 5 & 5 & 4 & 5 & 5 \\
\hline
\end{tabular}

\subsection{Algorithm}

The system algorithm is an explanation of the steps for solving problems in the design of a decision support system in Defining A Content Creator. This is done to increase the effective and efficient assessment. The following is a Flowchart of the WASPAS algorithm, which is as follows: 


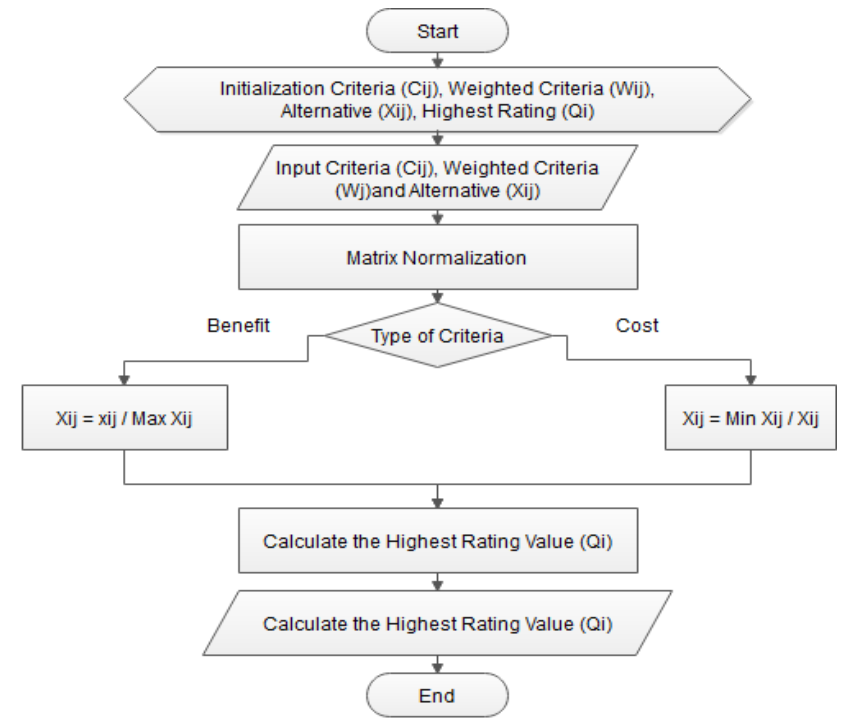

Figure 1. Flowchart of the WASPAS Algorithm

\subsection{Analysis and Result}

The following are the steps in completing the WASPAS method as follows:

1. Creating a Decision Matrix

The following is a decision matrix based on the data converted to alternative values, namely, as follows:

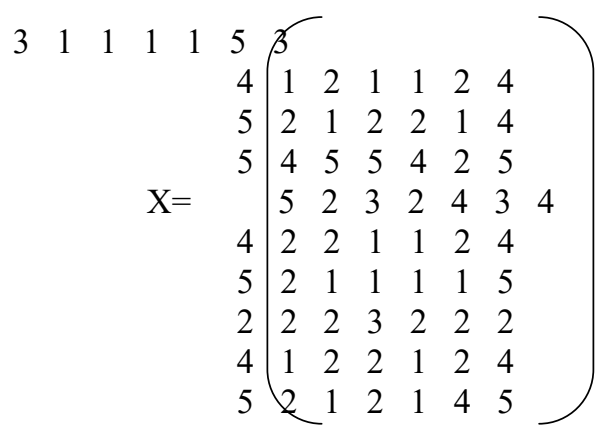

2. Calculating the Normalized Matrix

The following is a matrix normalization of alternative values according to the criteria.

$$
=\frac{x_{i j}}{\max i x_{i j}}
$$

Performance Criteria Matrix I:

$$
\begin{aligned}
& \mathrm{A}_{11}=\frac{3}{5}=0,6 \\
& \mathrm{~A}_{21}=\frac{4}{5}=0,8 \\
& \mathrm{~A}_{31}=\frac{5}{5}=1 \\
& \mathrm{~A}_{41}=\frac{5}{5}=1 \\
& \mathrm{~A}_{51}=\frac{5}{5}=1
\end{aligned}
$$

$$
\begin{aligned}
& \mathrm{A}_{61}=\frac{4}{5}=0,8 \\
& \mathrm{~A}_{71}=\frac{5}{5}=1 \\
& \mathrm{~A}_{81}=\frac{2}{5}=0,4 \\
& \mathrm{~A}_{91}=\frac{4}{5}=0,8 \\
& \mathrm{~A}_{101}=\frac{5}{5}=1
\end{aligned}
$$

Performance Criteria Matrix II :

$$
\begin{aligned}
& \mathrm{A}_{12}=\frac{1}{4}=0,25 \\
& \mathrm{~A}_{22}=\frac{1}{4}=0,25 \\
& \mathrm{~A}_{32}=\frac{2}{4}=0,5 \\
& \mathrm{~A}_{42}=\frac{4}{4}=1 \\
& \mathrm{~A}_{52}=\frac{2}{4}=0,5
\end{aligned}
$$

$$
\begin{aligned}
& \mathrm{A}_{62}=\frac{2}{4}=0,5 \\
& \mathrm{~A}_{72}=\frac{2}{4}=0,5 \\
& \mathrm{~A}_{82}=\frac{2}{4}=0,5 \\
& \mathrm{~A}_{92}=\frac{1}{4}=0,25 \\
& \mathrm{~A}_{102}=\frac{2}{4}=0,5
\end{aligned}
$$


Performance Criteria Matrix III :

$\mathrm{A}_{13}=\frac{1}{5}=0,2$
$\mathrm{~A}_{23}=\frac{2}{5}=0,4$
$\mathrm{~A}_{33}=\frac{1}{5}=0,2$
$\mathrm{~A}_{43}=\frac{5}{5}=1$
$\mathrm{~A}_{53}=\frac{3}{5}=0,6$

Performance Criteria Matrix IV :

$\mathrm{A}_{14}=\frac{1}{5}=0,2$
$\mathrm{~A}_{24}=\frac{1}{5}=0,2$
$\mathrm{~A}_{34}=\frac{2}{5}=0,4$
$\mathrm{~A}_{44}=\frac{5}{5}=1$
$\mathrm{~A}_{54}=\frac{2}{5}=0,4$

Performance Criteria Matrix V :

$$
\begin{aligned}
& \mathrm{A}_{15}=\frac{1}{4}=0,25 \\
& \mathrm{~A}_{25}=\frac{1}{4}=0,25 \\
& \mathrm{~A}_{35}=\frac{2}{4}=0,5 \\
& \mathrm{~A}_{45}=\frac{4}{4}=1 \\
& \mathrm{~A}_{55}=\frac{4}{4}=1
\end{aligned}
$$

$$
\begin{aligned}
& \mathrm{A}_{63}=\frac{2}{5}=0,4 \\
& \mathrm{~A}_{73}=\frac{1}{5}=0,2 \\
& \mathrm{~A}_{83}=\frac{2}{5}=0,4 \\
& \mathrm{~A}_{93}=\frac{2}{5}=0,4 \\
& \mathrm{~A}_{103}=\frac{1}{5}=0,2
\end{aligned}
$$

$\mathrm{A}_{64}=\frac{1}{5}=0,2$

$\mathrm{A}_{74}=\frac{1}{5}=0,2$

$\mathrm{A}_{84}=\frac{3}{5}=0,6$

$\mathrm{A}_{94}=\frac{2}{5}=0,4$

$\mathrm{A}_{104}=\frac{2}{5}=0,4$
$\mathrm{A}_{65}=\frac{1}{4}=0,25$
$\mathrm{A}_{75}=\frac{1}{4}=0,25$
$\mathrm{A}_{85}=\frac{2}{4}=0,5$
$\mathrm{A}_{95}=\frac{1}{4}=0,25$
$\mathrm{A}_{105}=\frac{1}{4}=0,25$

Performance Criteria Matrix VI :

$$
\begin{aligned}
& \mathrm{A}_{16}=\frac{5}{5}=1 \\
& \mathrm{~A}_{26}=\frac{2}{5}=0,4 \\
& \mathrm{~A}_{36}=\frac{1}{5}=0,2 \\
& \mathrm{~A}_{46}=\frac{2}{5}=0,4 \\
& \mathrm{~A}_{56}=\frac{3}{5}=0,6
\end{aligned}
$$

$$
\begin{aligned}
& \mathrm{A}_{66}=\frac{2}{5}=0,4 \\
& \mathrm{~A}_{76}=\frac{1}{5}=0,2 \\
& \mathrm{~A}_{86}=\frac{2}{5}=0,4 \\
& \mathrm{~A}_{96}=\frac{2}{5}=0,4 \\
& \mathrm{~A}_{106}=\frac{4}{5}=0,8
\end{aligned}
$$

Performance Criteria Matrix VII :

$$
\begin{aligned}
& \mathrm{A}_{17}=\frac{3}{5}=0,6 \\
& \mathrm{~A}_{27}=\frac{4}{5}=0,8 \\
& \mathrm{~A}_{37}=\frac{4}{5}=0,8 \\
& \mathrm{~A}_{47}=\frac{5}{5}=1 \\
& \mathrm{~A}_{57}=\frac{4}{5}=0,8
\end{aligned}
$$

$$
\begin{aligned}
& \mathrm{A}_{67}=\frac{4}{5}=0,8 \\
& \mathrm{~A}_{77}=\frac{5}{5}=1 \\
& \mathrm{~A}_{87}=\frac{2}{5}=0,4 \\
& \mathrm{~A}_{97}=\frac{4}{5}=0,8 \\
& \mathrm{~A}_{107}=\frac{5}{5}=1
\end{aligned}
$$

From the above calculations, the following results from the normalized performance matrix:

\section{Calculating the Value of Qi}

$\left(\begin{array}{ccccccc}0,6 & 0,25 & 0,2 & 0,2 & 0,25 & 1 & 0,6 \\ 0,8 & 0,25 & 0,4 & 0,2 & 0,25 & 0,4 & 0,8 \\ 1 & 0,5 & 0,2 & 0,4 & 0,5 & 0,2 & 0,8 \\ 1 & 1 & 1 & 1 & 1 & 0,4 & 1 \\ 1 & 0,5 & 0,6 & 0,4 & 1 & 0,6 & 0,8 \\ 0,8 & 0,5 & 0,4 & 0,2 & 0,25 & 0,4 & 0,8 \\ 1 & 0,5 & 0,2 & 0,2 & 0,25 & 0,2 & 1 \\ 0,4 & 0,5 & 0,4 & 0,6 & 0,5 & 0,4 & 0,4 \\ 0,8 & 0,25 & 0,4 & 0,4 & 0,25 & 0,4 & 0,8 \\ 1 & 0,5 & 0,2 & 0,4 & 0,25 & 0,8 & 1\end{array}\right)$


$Q i=0,5 \sum_{j=1}^{n} X i j w+0,5 \prod_{j=1}^{n}(x i j)^{w j}$

Score Q1

$=0,5\left(\left(0,6^{*} 0,2\right)+(0,25 * 0,2)+(0,2 * 0,2)+(0,2 * 0,05)+(0,25 * 0,2)+(1 * 0,05)+(0,6 * 0,1)\right)+0,5\left(\left(0.6^{0,2}\right) *\right.$ $\left.\left(0.25^{0,2}\right) *\left(0,2^{0,2}\right) *\left(0,2^{0,05}\right) *\left(0,25^{0,2}\right) *\left(1^{0,05}\right) *\left(0,6^{0,1}\right)\right)=$

$=0,19+0.16476=0.35476$

Score Q2

$=0,5((0,8 * 0,2)+(0,25 * 0,2)+(0,4 * 0,2)+(0,2 * 0,05)+(0,25 * 0,2)+(0,4 * 0,05)+(0,8+0,1))+0,5\left(\left(0.8^{0,2}\right) *\right.$ $\left.\left(0.25^{0,2}\right) *\left(0,4^{0,2}\right) *\left(0,2^{0,05}\right) *\left(0,25^{0,2}\right) *\left(0,4^{0,05}\right) *\left(0,8^{0,1}\right)\right)$

$=0,225+0.19708=0.42208$

Score Q3

$=0,5((1 * 0,2)+(0,5 * 0,2)+(0,2 * 0,2)+(0,4 * 0,05)+(0,5 * 0,2)+(0,2 * 0,05)+(0,8+0,1))+0,5\left(1^{0,2}\right) *\left(0.5^{0,2}\right)$

$\left.*\left(0,2^{0,2}\right) *\left(0,4^{0,05}\right) *\left(0,5^{0,2}\right) *\left(0,2^{0,05}\right) *\left(0,8^{0,1}\right)\right)$

$=0,275+0.23672=0.51172$

Score Q4

$=0,5((1 * 0,2)+(1 * 0,2)+(1 * 0,2)+(1 * 0,05)+(1 * 0,2)+(0,4 * 0,05)+(1+0,1))+0,5\left(1^{0,2}\right) *\left(1^{0,2}\right) *\left(1^{0,2}\right) *$ $\left.\left(1^{0,05}\right) *\left(1^{0,2}\right) *\left(0,4^{0,05}\right) *\left(1^{0,1}\right)\right)$

$=0,485+0.47761=0.96261$

Score Q5

$=0,5\left((1 * 0,2)+\left(0,5^{*} 0,2\right)+\left(0,6^{*} 0,2\right)+\left(0,4^{*} 0,05\right)+(1 * 0,2)+\left(0,6^{*} 0,05\right)+(0,8+0,1)\right)+0,5\left(1^{0,2}\right) *\left(0,5^{0,2}\right) *$ $\left.\left(0,6^{0,2}\right) *\left(0,4^{0,05}\right) *\left(1^{0,2}\right) *\left(0,6^{0,05}\right) *\left(0,8^{0,1}\right)\right)$

$=0,375+0.35786=0.73286$

Score Q6

$=0,5((0,8 * 0,2)+(0,5 * 0,2)+(0,4 * 0,2)+(0,2 * 0,05)+(0,25 * 0,2)+(0,4 * 0,05)+(0,8+0,1))+0,5\left(0,8^{0,2}\right) *$ $\left.\left(0,5^{0,2}\right) *\left(0,4^{0,2}\right) *\left(0,2^{0,05}\right) *\left(0,25^{0,2}\right) *\left(0,4^{0,05}\right) *\left(0,8^{0,1}\right)\right)$

$=0,25+0.22638=0.47638$

Score Q7

$=0,5((1 * 0,2)+(0,5 * 0,2)+(0,2 * 0,2)+(0,2 * 0,05)+(0,25 * 0,2)+(0,2 * 0,05)+(1+0,1))+0,5\left(\left(1^{0,2}\right) *\left(0,5^{0,2}\right)\right.$

$\left.*\left(0,2^{0,2}\right) *\left(0,2^{0,05}\right) *\left(0,25^{0,2}\right) *\left(0,2^{0,05}\right) *\left(1^{0,1}\right)\right)$

$=0,255+0.20355=0.45855$

Score Q8

$=0,5((0,4 * 0,2)+(0,5 * 0,2)+(0,4 * 0,2)+(0,6 * 0,05)+(0,5 * 0,2)+(0,4 * 0,05)+(0,4+0,1))+0,5\left(\left(0,4^{0,2}\right) *\right.$ $\left.\left(0,5^{0,2}\right) *\left(0,4^{0,2}\right) *\left(0,6^{0,05}\right) *\left(0,5^{0,2}\right) *\left(0,4^{0,05}\right) *\left(0,4^{0,1}\right)\right)$

$=0,225+0.22315=0.44815$

Score Q9

$=0,5((0,8 * 0,2)+(0,25 * 0,2)+(0,4 * 0,2)+(0,4 * 0,05)+(0,25 * 0,2)+(0,4 * 0,05)+(0,8+0,1))+0,5\left(\left(0,8^{0,2}\right) *\right.$ $\left.\left(0,25^{0,2}\right) *\left(0,4^{0,2}\right) *\left(0,4^{0,05}\right) *\left(0,25^{0,2}\right) *\left(0,4^{0,05}\right) *\left(0,8^{0,1}\right)\right)$

$=0,23+0.20403=0.43403$

Score Q10

$=0,5\left((1 * 0,2)+(0,5 * 0,2)+(0,2 * 0,2)+(0,4 * 0,05)+(0,25 * 0,2)+(0,8 * 0,05)+(1+0)+0,,5\left(\left(1^{0,2}\right) *\left(0,0,5^{0,2}\right)\right.\right.$

$\left.*\left(0,2^{0,2}\right) *\left(0,4^{0,05}\right) *\left(0,25^{0,2}\right) *\left(0,8^{0,05}\right) *\left(1^{0,1}\right)\right)$

$=0,275+0.22585=0.50085$

4. Ranking

Based on the Qi value above, the following results and rankings from the Qi assessment are shown in table 11. as follows:

Table 11. Results of the WASPAS Method ranking

\begin{tabular}{clcc}
\hline No & \multicolumn{1}{c}{ Alternative } & Score Qi & Rangking \\
\hline 1 & Naufal Alfaqir & 0.96261 & 1 \\
\hline 2 & Apri Manullang & 0.73286 & 2 \\
\hline 3 & Andriansyah & 0.51172 & 3 \\
\hline 4 & Evi Ulina Sitorus & 0.50085 & 4 \\
\hline 5 & Sri Wahyuni & 0.47638 & 5 \\
\hline 6 & Saiful Silunglingga & 0.45855 & 6 \\
\hline 7 & Dedy Syaputra & 0.44815 & 7 \\
\hline \multirow{2}{*}{8} & Febbry Anto & & 8 \\
\hline 9 & Situmorang & 0.43403 & \\
\hline 10 & Andika Chamberlin & 0.42208 & 9 \\
\hline
\end{tabular}


From the results of the ranking above, the one who passed to become a Content Creator is rank 1, namely Naufal Alfaqir with a value of 0.96261 . if there is the same value at the time of calculating the value of Qi and has the same rank, then the decision is given to the company leadership to determine who is entitled to be accepted as an employee of the content creator

\section{CONCLUSION}

1. The WASPAS method in this study provides the best alternative results from the analysis carried out.

2. Selection of the right criteria in the analysis makes this research very accurate in determining the results.

3. In applying the WASPAS method, the selection of criteria is based on the level of importance, and gives weight to the alternatives to be selected.

4. Analysis of this method can be used in different cases by providing suitable criteria for the problem to be solved.

\section{REFERENCES}

1. Abdullah, L., \& Adawiyah, C. W. (2014). Simple Additive Weighting Methods of Multi criteria Decision Making and Applications: A Decade Review. International Journal of Information Processing and Management(IJIPM), 5(1), 39-49. http://link.springer.com/10.1007/s10764-012-9584-5

2. Al-hafiz, N. W. (2017). Sistem Pendukung Keputusan Penentukan Kredit Pemilikan Rumah Menerapkan Multi-Objective Optimization On The Basis Of Ratio Analysis (MOORA ). I, 306-309.

3. Amalia, V., Syamsuar, D., \& Atika, L. (2019). Komparasi Metode Wp Saw Dan Waspas Dalam Penentuan Penerima Beasiswa Pmdk. Jurnal Bina Komputer, 1(2), 122-132. https://doi.org/10.33557/binakomputer.v1i2.452

4. Daniel, E. S., Crawford Jackson, E. C., \& Westerman, D. K. (2018). The Influence of Social Media Influencers: Understanding Online Vaping Communities and Parasocial Interaction through the Lens of Taylor's Six-Segment Strategy Wheel. Journal of Interactive Advertising, 18(2), 96-109. https://doi.org/10.1080/15252019.2018.1488637

5. Gunawan, S. (2015). Sistem Pendukung Keputusan Pemilihan Guru Terbaik Pada Sma Negeri 2 Kutacane Dengan Menggunakan Metode Simple Additive Weighting (Saw). Pelita Informatika Budi Darma, 9(3), 143-148.

6. Handayani, M., \& Marpaung, N. (2018). Implementasi Metode Weight Aggregated Sum Product Assesment (Waspas) Dalam Pemilihan Kepala Laboratorium. Seminar Nasional Royal (SENAR) 2018 ISSN 2622-9986 (Cetak) STMIK Royal-AMIK Royal, ISSN 2622-6510 (Online), 9986(September), 253 258.

7. Handayani, M., Marpaung, N., Informasi, S., \& Royal, S. (2018). IMPLEMENTASI METODE WEIGHT AGGREGATED SUM PRODUCT ASSESMENT ( WASPAS) DALAM PEMILIHAN KEPALA LABORATORIUM. 9986(September).

8. Hossein Hojjati, S. M., \& Anvary, A. (2013). An integrated SAW, TOPSIS method for ranking the major lean practices based on four attributes. World Applied Sciences Journal, 28(11), 1862-1871. https://doi.org/10.5829/idosi.wasj.2013.28.11.2079

9. Hutagalung, S. Y., Pratiwi, F., \& Wijaya, I. (2018). Penerapan Metode Weighted Aggregated Sum Product Assesment ( WASPAS ) Dalam Keputusan Penerimaan Beasiswa. 148-151.

10. Indriani, D., \& Warnilah, A. I. (2019). Sistem Informasi Hafalan Terbaik Dalam Munaqosah Tahfizhul Qur'an Menggunakan Metode Ahp. EVOLUSI - Jurnal Sains Dan Manajemen, 7(1), 1-11. https://doi.org/10.31294/evolusi.v7i1.5454

11. Kusrini M.Kom. (n.d.). Konsep dan Aplikasi Sistem Pendukung Keputusan, \{2014]\}\{Yogyakarta\}:\{C.V ANDI OFFSET\}.

12. McShane, L., Pancer, E., \& Poole, M. (2019). The Influence of B to B Social Media Message Features on Brand Engagement: A Fluency Perspective. Journal of Business-to-Business Marketing, 26(1), 1-18. https://doi.org/10.1080/1051712X.2019.1565132

13. Nasyuha, A. H. (2019). Sistem Pendukung Keputusan Menentukan Pemberian Pinjaman Modal dengan Metode Multi Attribute Utility Theory. JURNAL MEDIA INFORMATIKA BUDIDARMA, 3(2). https://doi.org/10.30865/mib.v3i2.1093

14. Panjaitan, S. M., Manik, S. O., \& Fau, A. (2019). Sistem Pendukung Keputusan Dengan Menerapkan Metode WASPAS Untuk Menentukan Guru Bidang Kesiswaan. 614-619.

15. Purwokerto, U. M., Sari, D. R., Utami, E., \& Lutfi, E. T. (2014). Menentukan Dosen Berprestasi Mengunakan Metode Simple Additive Weighting ( Saw) Dan Copeland Score ( Studi Kasus : Universitas Muhammadiyah Purwokerto ). 15(1), 5-13.

16. Safrizal, Sitorus, V. M., \& Napitupulu, D. (2018). Sistem Pendukung Keputusan Pengangkatan Guru 
Tetap Menerapkan Metode Weight Aggregated Sum Product Assesment (WASPAS ). 2(2), 10-15.

17. Salman, A., Mustaffa, N., Mohd Salleh, M. A., \& Ali, M. N. S. (2016). Social media and agenda setting: Implications on political agenda. Jurnal Komunikasi: Malaysian Journal of Communication, 32(1), 607623. https://doi.org/10.17576/jkmjc-2016-3201-19

18. Setiani, B. (2013). Kajian Sumber Daya Manusia Dalam Proses Rekrutmen Tenaga Kerja Di Perusahaan. Jurnal Ilmiah Widya, 1(1), 38-44.

19. Sianturi, M., Tarigan, J., Rizanti, N. P., \& Cahyadi, A. D. (2018). Sistem Pengambilan Keputusan Pemilihan Jurusan Terbaik Pada Kuliah Menerapkan Metode Weight Aggregated Sum Product Assesment ( WASPAS ). Sensasi, 10(20), 160-164.

20. Sugiarti, S., Nahulae, D. K., Panggabean, T. E., \& Sianturi, M. (2018). Sistem Pendukung Keputusan Penentuan Kebijakan Strategi Promosi Kampus Dengan Metode Weighted Aggregated Sum Product Assesment ( WASPAS ). 5(2), 103-108.

21. Zavadskas, E. K., Vainiūnas, P., Turskis, Z., \& Tamošaitienè, J. (2012). Multiple criteria decision support system for assessment of projects managers in construction. International Journal of Information Technology and Decision Making, 11(2), 501-520. https://doi.org/10.1142/S0219622012400135 